\title{
Mechanisms of the Deleterious Effects of Tamoxifen on Mitochondrial Respiration Rate and Phosphorylation Efficiency
}

\author{
Carla M. P. Cardoso, ${ }^{*} \dagger$ José B. A. Custódio, ${ }^{*},{ }^{\dagger}, 1$ Leonor M. Almeida, ${ }^{*} \dagger$ and António J. M. Moreno $\dagger$ \\ *Laboratório de Bioquímica, Faculdade de Farmácia, and †Centro de Neurociências de Coimbra, Universidade de Coimbra, Coimbra, Portugal
}

Received January 10, 2001; accepted July 23, 2001

Mechanisms of the Deleterious Effects of Tamoxifen on Mitochondrial Respiration Rate and Phosphorylation Efficiency. Cardoso, C. M. P., C ustódio, J. B. A., Almeida, L. M., and Moreno, A. J. M. (2001). Toxicol. Appl. Pharmacol. 176, 145- 152.

Tamoxifen (TAM), the widely prescribed drug in the prevention and therapy of breast cancer, is a well-known modulator of estrogen receptor (ER) that also inhibits the proliferation of different cell types that lack the ER. However, the ER-independent action mechanisms of TAM and its side effects have not been yet clarified. Mitochondria are essential in supporting the energy-dependent regulation of cell functions. Changes in mitochondria result in bioenergetic deficits leading to the loss of vital functions to cell survival. Therefore, this study describes the effects of TAM on mitochondrial bioenergetics, contributing to a better understanding of the biochemical mechanisms underlying the multiple antiproliferative and toxic effects of this drug. TAM at concentrations above $20 \mathrm{nmol} / \mathrm{mg}$ protein, preincubated with isolated rat liver mitochondria at $25^{\circ} \mathrm{C}$ for $3 \mathrm{~min}$, significantly depresses, in a dose-dependent manner, the phosphorylation efficiency of mitochondria as inferred from the decrease in the respiratory control and ADP/O ratios, the perturbations in mitochondrial transmembrane potential $(\Delta \Psi)$, the fluctuations associated with mitochondrial energization, and the phosphorylative cycle induced by ADP. Furthermore, TAM at up to $40 \mathrm{nmol} / \mathrm{mg}$ protein stimulates the rate of state 4 respiration and at higher concentrations it strongly inhibits state 3 and uncouples the mitochondrial respiration. The stimulation of state 4 respiration parallels the decrease of $\Delta \Psi$ as a consequence of proton permeability. The TAM-stimulatory action of ATPase is also observed in intact mitochondria, suggesting that TAM promotes extensive permeability to protons due to destructive effects in the structural integrity of the mitochondrial inner membrane. These multiple effects of TAM on mitochondrial bioenergetic functions, causing changes in the respiration, phosphorylation efficiency, and membrane structure, may explain the cell death induced by this drug in different cell types, its anticancer activity in ER-negative cells, and its side effects. $\odot 2001$ Academic Press

Key Words: tamoxifen; anticancer drug; mitochondria; respiration rate; mitochondrial transmembrane potential; mitochondrial proton leak; membrane disruption.

${ }^{1}$ To whom correspondence should be addressed at Laboratório de Bioquímica, Faculdade de Farmácia, Couraça dos Apóstolos, 51, r/c, 3000-295 Coimbra, Portugal. Fax: +351-239-852569; E-mail: custodio@ci.uc.pt.
Tamoxifen (TAM) is the most used nonsteroidal antiestrogen drug for chemotherapy and chemoprevention of breast cancer (Neven and Vernaeve, 2000; Radmacher and Simon, 2000). The antiproliferative effects of TAM in estrogen-dependent breast cancer cells are mediated by high-affinity binding to the estrogen receptor (ER) (Coezy et al., 1982). However, TAM inhibits also the growth of ER-negative breast cancer cells and other cell types that lack ER (Couldwell et al., 1993; Croxtall et al., 1994; Charlier et al., 1995). Actually, TAM has been reported to have several physiological effects that are ER independent, including sensitization of resistant tumor cells to many chemotherapeutic agents (Altan et al., 1999) and several pleiotropic effects both in vivo and in vitro (Chen et al., 1999 and references therein). Moreover, it has been reported that TAM induces multiple cellular adverse effects, including hemolytic action (Suwalsky et al., 1998; Cruz Silva et al., 2000) and inhibition of mitochondrial permeability transition (Custódio et al., 1998). However, the multiple mechanisms underlying the TAM-induced cytotoxic effects have not been fully understood and the identification of such mechanisms is relevant to assure a safe and appropriate therapeutic administration of this drug.

The effects of TAM on a variety of biological membranes have been reported to be a consequence of its biophysical and biochemical interactions with biomembranes (Custódio et al., 1993, 1996) that are related with its strong partition within the lipid bilayer (Custódio et al., 1991). Such effects include the stimulation of ATP hydrolysis (Custódio et al., 1996; Chen et al., 1999) and the decrease in the energetic efficiency of the $\mathrm{Ca}^{2+}$ pump of sarcoplasmic reticulum (Custódio et al., 1996), modifications in the morphology and structure of the breast tumor cell membranes, potentially responsible for its estrogenindependent antiproliferative activity (Sica et al., 1984), hemolytic effects (Cruz Silva et al., 2000), and mitochondrial swelling (Custódio et al., 1998).

Mitochondria are essential to support the energy-dependent regulation of several cell functions. The multifaceted role of mitochondria in cell homeostasis is rooted in the proton motive force $(\Delta p)$, which supplies the energy required for cell sustenance. In addition to ATP synthesis, mitochondria are also critical to the modulation of cell osmotic regulation, redox 
status, $\mathrm{pH}$ control, cell signal transduction, and $\mathrm{Ca}^{2+}$ homeostasis. Disruption of mitochondrial bioenergetics has been recognized to participate in the lethal cell injury induced by xenobiotics, leading to cellular ATP depletion and cell death (Wallace et al., 1997). Therefore, studies of TAM on the bioenergetic functions of mitochondria will contribute toward a better understanding of the biochemical mechanisms underlying its ER-independent actions and toward predicting its side effects. The results clearly demonstrate that TAM affects mitochondrial bioenergetics by disrupting the mitochondrial membrane, suggesting that such damage may be responsible for the demise of the TAM-induced cell.

\section{MATERIALS AND METHODS}

Chemicals. Tamoxifen, bovine serum albumin (BSA), Hepes, EDTA, nigericin, and oligomycin were purchased from Sigma Chemical Co. (St. Louis, MO). Carbonyl cyanide $p$-trifluoromethoxyphenylhydrazone (FCCP), EGTA, tetraphenylphosphonium $\left(\mathrm{TPP}^{+}\right)$, and sucrose were from Merck (Darmstadt, Germany). All other chemicals were commercial products of the highest purity grade available. Solutions were prepared in deionized ultrapure water.

Isolation of rat liver mitochondria. Mitochondria were prepared from Wistar rats $(250-300 \mathrm{~g})$ by differential centrifugation as described elsewhere (Moreno and Madeira, 1991), with slight modifications. Rats were killed by decapitation and the liver was removed, finely minced, and washed in ice-cold isolation medium containing $250 \mathrm{mM}$ sucrose, $0.5 \mathrm{mM}$ EGTA, $0.5 \mathrm{mM}$ EDTA, $10 \mathrm{mM}$ Hepes-KOH, pH 7.4, and $0.1 \%(\mathrm{w} / \mathrm{v})$ free fatty acid BSA. Tissue fragments were quickly homogenized with a motor-driven Teflon Potter homogenizer in the presence of ice-cold isolation medium $(7 \mathrm{~g} / 50 \mathrm{ml})$. Liver homogenate was centrifuged at $800 \mathrm{~g}$ for $10 \mathrm{~min}$ (IEC B-20A centrifuge) at $4^{\circ} \mathrm{C}$ and mitochondria were recovered from the supernatant by centrifugation at $10,000 \mathrm{~g}$ for $10 \mathrm{~min}$. The mitochondrial pellet was resuspended using a paintbrush and centrifuged twice at $10,000 \mathrm{~g}$ for $10 \mathrm{~min}$ before obtaining a final mitochondrial suspension. EGTA and BSA were omitted from the final washing medium, which was adjusted to $\mathrm{pH}$ 7.2. Mitochondrial protein content was determined by the Biuret method (Gornall et al., 1949) using BSA as the protein standard.

Measurement of oxygen consumption. Mitochondrial oxygen consumption (respiration rate) was monitored polarographically at $25^{\circ} \mathrm{C}$ using a Clarktype oxygen electrode (YSI Model 5331, Yellow Spring Instruments) connected to a suitable recorder in a 1-ml thermostated, water-jacketed, sealed glass chamber with constant magnetic stirring, as described elsewhere (Custódio et al., 1994; Ferreira et al., 1997). Mitochondria at a concentration of 1 $\mathrm{mg}$ of protein/ml were suspended in a standard respiratory medium containing $130 \mathrm{mM}$ sucrose, $50 \mathrm{mM} \mathrm{KCl}, 5 \mathrm{mM} \mathrm{MgCl}, 0.1 \mathrm{mM}$ EGTA, $5 \mathrm{mM} \mathrm{KH} \mathrm{KO}_{4}$,

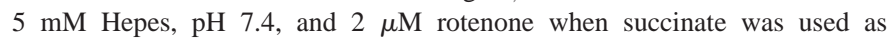
substrate. State 4 respiration was initiated upon addition of $5 \mathrm{mM}$ succinate and ADP was added to establish state 3 respiration momentarily $(150 \mu \mathrm{M})$ or permanently $(1.5 \mathrm{mM})$. Respiratory control ratios $(\mathrm{RCR}=$ state $3 /$ state 4$)$, respiratory states, and ADP/O ratios were determined according to Chance and Williams (1956). TAM was added in ethanolic solutions (up to $6 \mu \mathrm{l}$ ) to the reaction medium with mitochondria and allowed to incubate for 3 min before starting the reactions. This incubation period was carried out to ensure the complete internalization of the compound on the membrane due to its lipophilic characteristic (Custódio et al., 1991). Care was taken to ensure a final assay volume of $1 \mathrm{ml}$ after the additions. The scale of oxygen uptake was calibrated according to the oxygen consumed by submitochondrial particles after addition of titrated solutions of NADH.

Mitochondrial membrane potential. Mitochondrial membrane potential $(\Delta \Psi)$ was estimated with an ion-selective electrode of $\mathrm{TPP}^{+}$prepared accord- ing to Kamo et al. (1979) to measure the transmembrane distribution of $\mathrm{TPP}^{+}$ as previously established (Kamo et al., 1979; Moreno and Madeira, 1991), using an $\mathrm{Ag} / \mathrm{AgCl}$-saturated electrode as reference (Model MI 402; Microelectrodes, Inc., Bedford, $\mathrm{NH}$ ). The reactions were carried out in an open vessel with magnetic stirring at $25^{\circ} \mathrm{C}$ and performed with $1 \mathrm{mg}$ mitochondrial protein in $2 \mathrm{ml}$ of the standard respiratory medium supplemented with $2 \mu \mathrm{M}$ rotenone and $4 \mu \mathrm{M} \mathrm{TPP}{ }^{+}$. TAM was added in ethanolic solutions (up to $6 \mu \mathrm{l}$ ) to the standard respiratory medium after protein addition and incubated for $3 \mathrm{~min}$ before starting the reactions. The experiments were started by adding $5 \mathrm{mM}$ succinate and, after a steady-state distribution of $\mathrm{TPP}^{+}$(ca. 2 min of recording), $150 \mathrm{nmol} / \mathrm{mg}$ protein ADP was added. Control assays were conducted in the presence of an identical volume of ethanol to that used in the experiments with TAM. $\Delta \Psi$ is expressed in $\mathrm{mV}$ and was estimated from the decrease of $\mathrm{TPP}^{+}$concentration in the reaction medium, as described elsewhere (Moreno and Madeira, 1991). Preliminary calibrations in the presence of TAM revealed no direct interference with the electrode signal.

Simultaneous measurements of respiration rate and $\Delta \boldsymbol{\Psi}$. To determine the TAM effects on mitochondrial function and to discriminate its site of action on mitochondria, we adopted the approach commonly used for top-down metabolic control analysis (Hafner et al., 1990). In this approach, oxidative phosphorylation is conceptually divided into three subsystems: the mitochondrial respiratory chain, the phosphorylation system, and the proton leak. The dependence of each of these three subsystems on their common intermediatethe proton motive force $-\Delta \mathrm{p}$ is measured in either the presence or the absence of the compounds in order to determine which of these subsystems is directly affected.

To assess the effects of TAM on the $\Delta$ p producing system (i.e., the respiratory chain complexes and the substrate transporters) mitochondrial respiration and $\Delta \Psi$ were measured simultaneously at $25^{\circ} \mathrm{C}$ in a $1-\mathrm{ml}$ incubation chamber fitted with both a Clark-type oxygen electrode and a $\mathrm{TPP}^{+}$sensitive electrode. Mitochondria $(1 \mathrm{mg})$ were suspended in the reaction medium containing $130 \mathrm{mM}$ sucrose, $50 \mathrm{mM} \mathrm{KCl}, 5 \mathrm{mM} \mathrm{MgCl}_{2}, 0.1 \mathrm{mM}$ EGTA, $5 \mathrm{mM} \mathrm{KH} \mathrm{PO}_{4}, 5 \mathrm{mM}$ Hepes, $\mathrm{pH} 7.4$, supplemented with $3 \mu \mathrm{M}$ rotenone, $4 \mu \mathrm{M} \mathrm{TPP}^{+}, 1.5 \mu \mathrm{g}$ oligomycin (to eliminate phosphorylation), and $50 \mathrm{ng}$ nigericin [nigericin eliminates the $\mathrm{pH}$ gradient across the mitochondrial inner membrane (Murphy and Brand, 1987; Hafner and Brand, 1991) and therefore $\Delta \Psi$ becomes equal to $\Delta \mathrm{p}]$. Mitochondria were incubated under these conditions for $3 \mathrm{~min}$ in the presence of TAM. Respiration was initiated by the addition of $5 \mathrm{mM}$ potassium succinate $(\mathrm{pH} 7.2)$. Respiration and $\Delta \Psi$ were titrated by successive additions of FCCP (up to $40 \mathrm{nM}$ ). At the end of the incubation, FCCP $(0.35 \mu \mathrm{M})$ was added to allow the $\mathrm{TPP}^{+}$electrode to return to its baseline.

The previous protocol was followed to assess the effects of TAM on proton leak (i.e., the passive permeability of the mitochondrial inner membrane to protons, and any cation cycling reactions), but the titration was done by successive additions of malonate (up to $1.5 \mathrm{mM}$ ).

Titrations with TAM were conducted under the same conditions but in the absence of FCCP or malonate.

ATPase activity of intact mitochondria. ATPase activity was estimated by monitoring the changes in $\mathrm{pH}$ of the medium associated with ATP hydrolysis (Madeira et al., 1974). The experiments were carried out at $25^{\circ} \mathrm{C}$ in $2 \mathrm{ml}$ of the standard respiratory medium lightly buffered with $0.5 \mathrm{mM}$ Hepes, which was adjusted to $\mathrm{pH} 7.2$ and supplemented with $2 \mu \mathrm{M}$ rotenone. The reactions were performed in $1 \mathrm{mg}$ mitochondrial protein and initiated with $2 \mathrm{mM} \mathrm{Mg}-\mathrm{ATP}$ as described elsewhere (Ferreira et al., 1997). TAM was incubated with mitochondria for $3 \mathrm{~min}$ before starting the reactions. The addition of $2 \mu \mathrm{g} / \mathrm{mg}$ protein oligomycin completely abolished the $\mathrm{pH}$ changes.

The results shown represent typical recordings from experiments of at least three different mitochondrial preparations.

\section{RESULTS}

To elucidate TAM effects on rat liver mitochondrial energetic metabolism, we studied the respiration parameters as well 


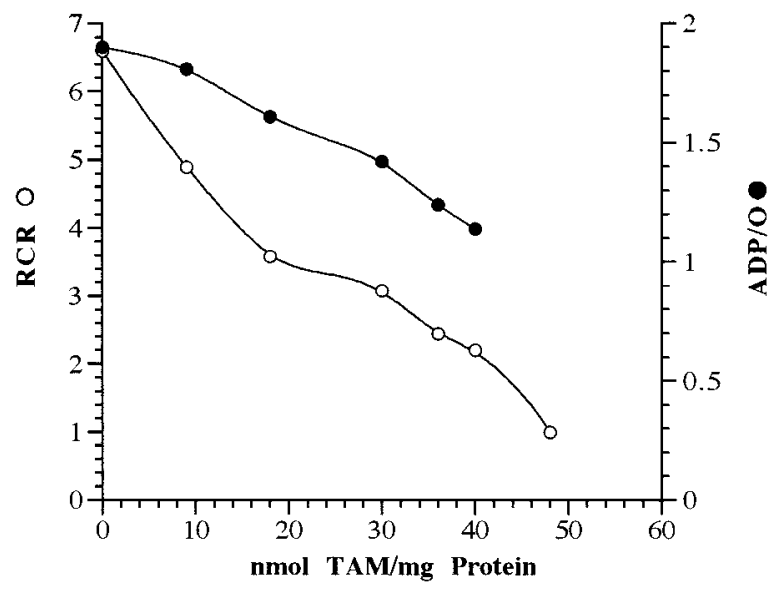

FIG. 1. Effects of TAM on mitochondrial RCR (O) and ADP/O (•) ratios. Mitochondria $(1 \mathrm{mg})$ in $1 \mathrm{ml}$ of the standard respiratory medium supplemented with $2 \mu \mathrm{M}$ rotenone were incubated with TAM for 3 min prior addition of succinate (state 4). After 2 min of energization, mitochondria were supplemented with ADP $(150 \mathrm{nmol} / \mathrm{mg}$ protein) to induce a state 3 condition. Mitochondrial respiration rates were determined by $\mathrm{O}_{2}$ consumption using a Clark-type electrode. Both indexes were calculated as described under Materials and Methods.

as the $\Delta \Psi$ of energized mitochondria. As shown in Fig. 1, at increasing concentrations, using succinate as respiratory substrate, TAM progressively depressed the RCR and the phosphorylative index (ADP/O ratio) of liver mitochondria. These results suggest a depressive effect on the phosphorylation capacity of mitochondria. RCR is a measure of the dependence of the respiratory rate on ADP; it indicates the ratio between the oxygen consumption rate in the presence of added ADP (state 3 ) and the rate obtained after the completion of phosphorylation pulse (state 4). The efficiency of mitochondrial oxidative phosphorylation is defined by the ADP/O ratio, i.e., the ratio between the nanomoles of added ADP and the nanograms of oxygen atoms consumed during the phosphorylation pulse. The ADP/O ratios were measured up to $40 \mathrm{nmol}$ $\mathrm{TAM} / \mathrm{mg}$ mitochondrial protein; for higher proportions of the drug, RCR approaches 1.0, meaning that ADP does not induce stimulation of respiratory rate. The depressive effect of TAM on the phosphorylation capacity of mitochondria was further confirmed by following the $\Delta \Psi$ fluctuations associated with mitochondrial respiration and with the phosphorylation cycle induced by ADP (Fig. 2). When succinate was added, mitochondria immediately developed a $\Delta \Psi$ of about $220 \mathrm{mV}$ (negative inside). Upon ADP addition (150 nmol/mg protein), which induces transition to state 3 , the $\Delta \Psi$ suffered an immediate fall to $180 \mathrm{mV}$, since ATP-synthase makes use of $\Delta \Psi$ to phosphorylate the added ADP, and, after a short lag phase, the mitochondrial membrane was repolarized close to its initial value (state 4). However, when TAM was present, mitochondria developed a consistently lower $\Delta \Psi$, although it was not very noticeable at up to $20 \mathrm{nmol} \mathrm{TAM} / \mathrm{mg}$ protein. Furthermore, the depolarization following the addition of ADP and the rate of repolarization on recovery from state 3 were progressively lower at increasing concentrations of TAM. These effects became very significant at $40 \mathrm{nmol} \mathrm{TAM} / \mathrm{mg}$ protein. The

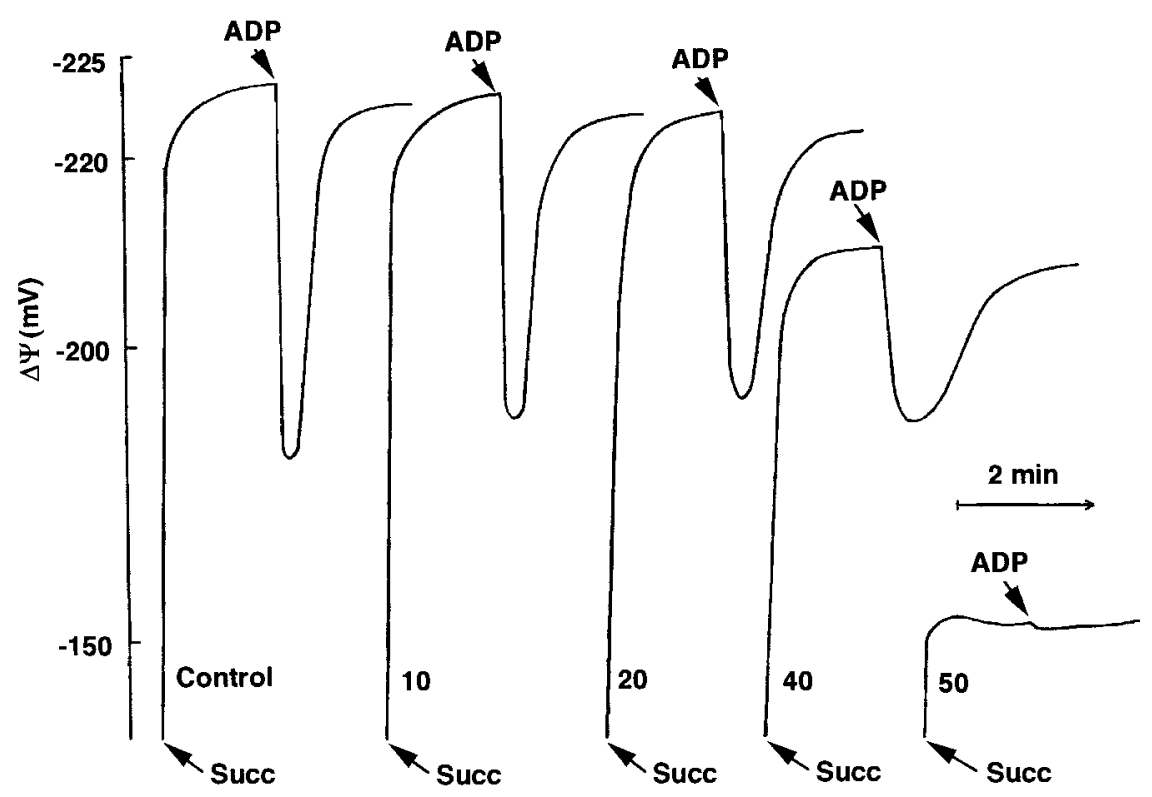

FIG . 2. Effect of TAM on the mitochondrial transmembrane potential $(\Delta \Psi)$. Mitochondria $(1 \mathrm{mg})$ suspended in $1 \mathrm{ml}$ of the standard respiratory medium supplemented with $4 \mu \mathrm{M} \mathrm{TPP}{ }^{+}$and $2 \mu \mathrm{M}$ rotenone were energized with $5 \mathrm{mM}$ succinate (succ) after incubation at $25^{\circ} \mathrm{C}$ for 3 min in the absence (control) and in the presence of different TAM concentrations (nmol TAM/mg protein) as indicated by the numbers adjacent to the traces. ADP (150 $\mathrm{nmol} / \mathrm{mg}$ protein) was added after a steady-state distribution of $\mathrm{TPP}^{+}$to induce state 3 respiration. $\Delta \Psi$ was determined using a $\mathrm{TPP}^{+}$-sensitive electrode as described under Materials and Methods. 


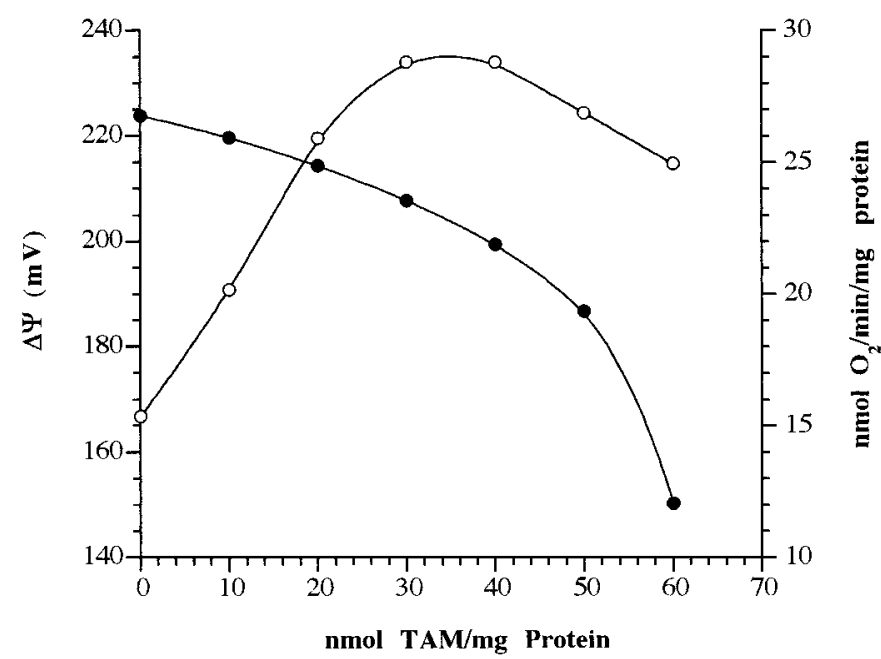

FIG. 3. Effects of sequential additions of TAM on $\Delta \Psi(\bullet)$ and state 4 respiration $(\bigcirc)$ of nonphosphorylating mitochondria. Mitochondria (1 mg) were suspended in $1 \mathrm{ml}$ of the standard respiratory medium supplemented with $50 \mathrm{ng} / \mathrm{ml}$ nigericin, $1.5 \mu \mathrm{g} / \mathrm{ml}$ oligomycin, $2 \mu \mathrm{M}$ rotenone, $4 \mu \mathrm{M} \mathrm{TPP}^{+}$and were energized with $5 \mathrm{mM}$ succinate. Mitochondrial respiration rates $(\bigcirc)$ and $\Delta \Psi(\bullet)$ were evaluated simultaneously with a Clark-type $\mathrm{O}_{2}$ electrode and a $\mathrm{TPP}^{+}$selective electrode, respectively, placed in the same closed reaction chamber. After a steady-state distribution of $\mathrm{TPP}^{+}$, the mitochondria suspension was titrated with sequential additions, at 2-min intervals, of $10 \mathrm{nmol}$ $\mathrm{TAM} / \mathrm{mg}$ protein.

lower steady state of $\Delta \Psi$ resulting from ADP additions is caused by the phosphorylation process (Kamo et al., 1979). The time length of this lower steady state is in fact related to the amount of added ADP and the $\Delta \Psi$ restoration to the original values is dependent on the coupling conditions of the system. Actually, the curves in Fig. 2 indicate that TAM has an uncoupling action, in agreement with the significant induced decrease in the RCR and ADP/O ratios, as shown in Fig. 1.

At the concentration of $50 \mathrm{nmol} \mathrm{TAM} / \mathrm{mg}$ protein $(50 \mu \mathrm{M})$, the drug induced a drastic depolarization of $\Delta \Psi$ and mitochondria were unable to phosphorylate the added ADP (i.e., RCR = 1, see Fig. 1). The TAM/protein ratio that induced this effect is in the narrow range of 50-60 nmol TAM/mg protein, depending on the mitochondrial preparation.

The depolarization of $\Delta \Psi$ induced by TAM was further provided by the sequential addition of TAM to mitochondria (Fig. 3). At concentrations up to $40 \mathrm{nmol} \mathrm{TAM} / \mathrm{mg}$ protein, the titration of mitochondria with TAM caused a progressive decrease in $\Delta \Psi$ in parallel with an increase in the state 4 respiration. At higher concentrations of TAM, the initial stimulating effect was followed by a progressive decrease in the respiratory rate stimulation, while mitochondrial $\Delta \Psi$ sharply decreased. This biphasic behavior indicates the existence of at least two effects or a pleiotropic action of the drug. The stimulation of state 4 respiration suggests that TAM increases the proton leak through the mitochondrial inner membrane and the decrease in stimulation observed at higher concentrations of TAM is probably caused by the inhibition of the respiratory chain compo- nents. This hypothesis can be conveniently checked out by studying the effect of the drug on the uncoupled respiration. Under these conditions, variations in membrane permeability do not interfere with the inhibition of the respiratory chain, because the permeability is always maximum. Therefore, to clarify the effects of TAM on mitochondria, we also studied the effects of TAM under either the state 3 conditions (in the presence of $1.5 \mathrm{mM} \mathrm{ADP}$ ) or the uncoupled conditions (in the presence of $1 \mu \mathrm{M}$ FCCP) (Fig. 4). Both the ADP-stimulated respiration and the FCCP (uncoupled) respiration were inhibited by TAM. The effect of TAM on FCCP-induced respiration reflects its interaction with the mitochondrial redox chain and confirms the impairment in the electron transfer along the respiratory chain. Additionally, the state 3 respiration is more sensitive to inhibition by TAM than the uncoupled respiration.

In agreement with the stimulatory effect of TAM on state 4 respiration, indicative of an increase in the proton leak through the mitochondrial inner membrane, TAM consistently increased the ATPase activity of tightly coupled intact mitochondria (Fig. 5). This effect reinforces the action mechanism of TAM as uncoupler, taking into account that its stimulatory activity is typical of an uncoupler or uncoupling substance, as observed for FCCP (Fig. 5). It is noteworthy that high concentrations of TAM $(>50 \mathrm{nmol} \mathrm{TAM} / \mathrm{mg}$ protein) induced a stimulation of the ATPase activity greater than that promoted by FCCP, possibly due to some kind of putative disruption effects on mitochondrial inner membrane. Moreover, while the stimulation of ATPase activity promoted by FCCP is abolished by oligomycin, the same induced by TAM is slightly depressed

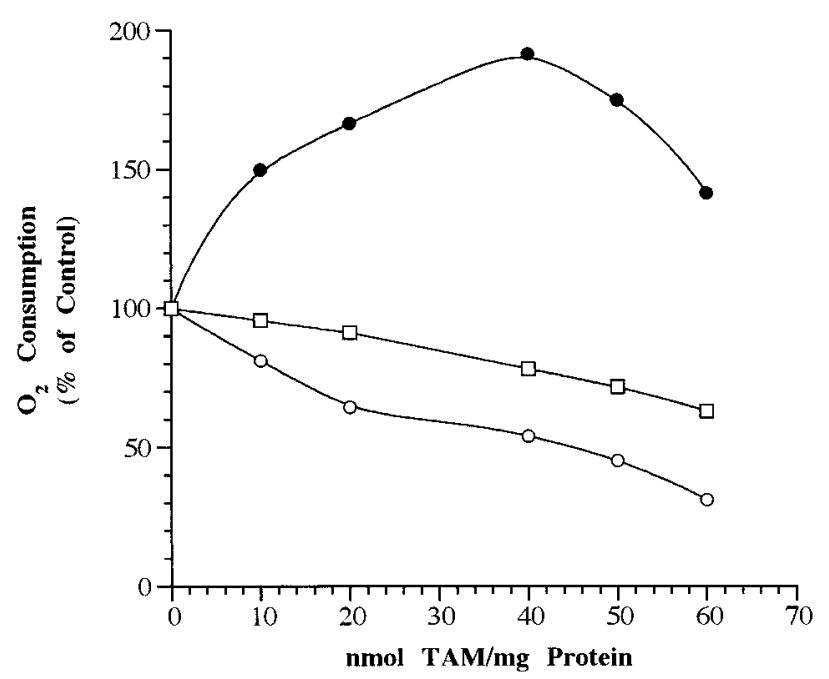

FIG . 4. Effects of TAM on state $4(\bullet)$, state $3(\bigcirc)$, and FCCP(uncoupled)respiration $(\square)$. Mitochondria $(1 \mathrm{mg}$ ) were incubated with TAM in $1 \mathrm{ml}$ of the respiratory standard medium at $25^{\circ} \mathrm{C} / 3$ min prior energization with $5 \mathrm{mM}$ succinate. The ADP- and FCCP-stimulated respiration were initiated by the addition of $1.5 \mathrm{mM}$ ADP $(\bigcirc)$ and $1.5 \mu \mathrm{M}$ FCCP $(\square)$, respectively, 2 min after the energization of mitochondria. Mitochondrial respiration rates were determined by $\mathrm{O}_{2}$ consumption using a Clark-type electrode and expressed as percentage of control (mitochondria in the absence of TAM). 


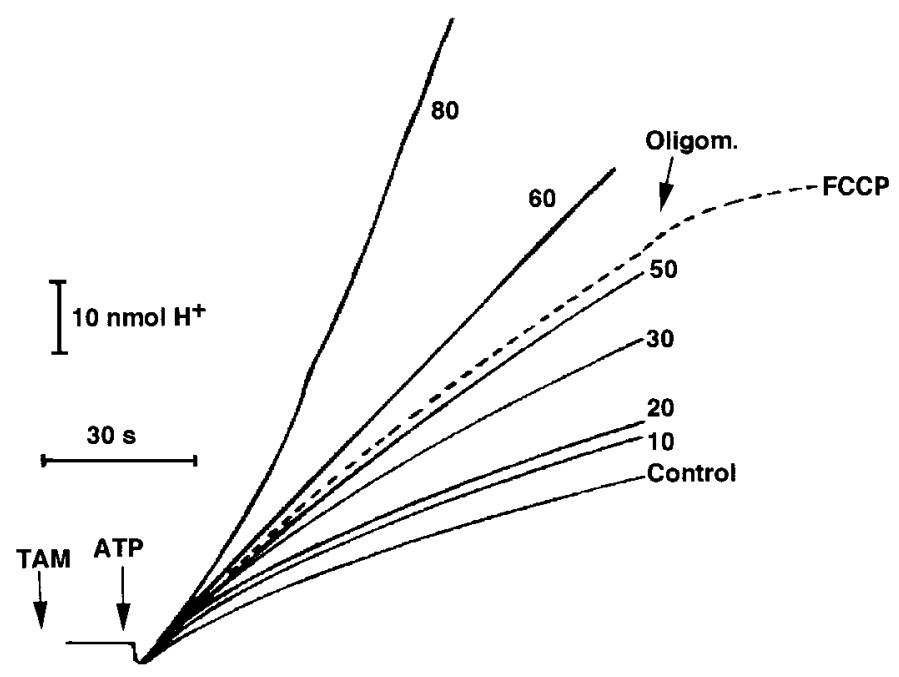

FIG. 5. Effects of TAM (-) and FCCP (-- ) on ATPase activity of intact mitochondria. The ATPase activity was evaluated at $25^{\circ} \mathrm{C}$ in $2 \mathrm{ml}$ of medium containing $130 \mathrm{mM}$ sucrose, $50 \mathrm{mM} \mathrm{KCl}, 2.5 \mathrm{mM} \mathrm{MgCl}_{2}, 5 \mathrm{mM}$ $\mathrm{KH}_{2} \mathrm{PO}_{4}, 0.5 \mathrm{mM}$ Hepes, $0.1 \mathrm{mM}$ EGTA, pH 7.2, and supplemented with 2 $\mu \mathrm{M}$ rotenone. After incubation of $1 \mathrm{mg}$ mitochondria for $3 \mathrm{~min}$ with different concentrations of TAM (nmol/mg protein), as indicated by the numbers adjacent to the traces, the reactions were started by adding $2 \mathrm{mM} \mathrm{Mg-ATP}$ and monitored by following the production of protons with a $\mathrm{pH}$ electrode. Oligomycin $(2 \mu \mathrm{g} / \mathrm{mg}$ protein) was added $2 \mathrm{~min}$ after $\mathrm{Mg}$-ATP addition.

and the ATPase of submitochondrial particles is not significantly affected by TAM (results not shown), suggesting disruption effects on mitochondrial inner membrane, as inferred from the depolarization of $\Delta \Psi$ (Fig. 2) and from the permeabilization to protons induced by TAM (Figs. 3 and 4).

To confirm our results, additional experiments were carried out in order to directly investigate the TAM action sites responsible for the observed effects on $\Delta \Psi$ and respiratory rates. We adopted the approach developed by Brand (1990) that is ideally suited to analyze compounds with pleiotropic effects on oxidative phosphorylation and has been used to investigate the mechanism of thyroid hormones (Hafner et al., 1988, 1989, 1990), glucagon (Brand et al., 1990), butylated hydroxyanisole (Fusi et al., 1992), and DDE (Ferreira et al., 1997) and to study functional variations in the liver mitochondria compartment in 25- and 60-day-old rats (Lionetti et al., 1998). The putative proton leaks induced by TAM through the mitochondrial inner membrane were investigated in nonphosphorylating mitochondria titrated with malonate, a respiratory inhibitor, and $\Delta \Psi$ ( $\Delta \Psi$ equals $\Delta \mathrm{p}$ in the used experimental conditions) was plotted against the respiration rate (Fig. 6). In the steady state, the proton efflux must be equal to the proton leak, assuming that no slip in the proton pumps occurs (Murphy, 1989). In addition, any secondary effect on the leak due to alterations in the $\Delta \mathrm{p}$ value is eliminated. Therefore, if a given compound increases the proton leak across the mitochondrial inner membrane, the curve will be displaced downward and to the right. As we can observe in Fig. 6 , at any given value of $\Delta \Psi$, the respiration is greater in the presence of TAM and a shift in this curve occurs downward and to the right that, according to previous reports (Brand, 1990; Fusi et al., 1992; Lionetti et al., 1998), suggests an increase in the proton leak through the mitochondrial inner membrane induced by this drug.

Figure 7 shows the titration of nonphosphorylating mitochondria with an uncoupler (FCCP) to increase the rate of respiration and to decrease $\Delta \Psi$. In this case, the obtained plot is a description of the kinetic response of the $\Delta$ p producers to their product, $\Delta \mathrm{p}$. Consequently, when a compound inhibits any component of the $\Delta \mathrm{p}$ producing system, the curve will be displaced downward and to the left, e.g., at a given value of $\Delta \mathrm{p}$, the respiration rate will decrease. In Fig. 7, the presence of TAM displaces the titration of $\Delta \Psi$ against respiration rate downward and to the left. Therefore, TAM inhibits one or more components of the $\Delta \mathrm{p}$ generating system (i.e., the electron transport system and the substrate transporters).

\section{DISCUSSION}

This work demonstrates that TAM has various effects on liver mitochondria. TAM preincubated (Fig. 2) or added sequentially to mitochondria (Fig. 3) causes depolarization of $\Delta \Psi$, stimulates the rate of state 4 respiration (Figs. 3 and 4 ), and inhibits the rate of state 3 respiration stimulated by ADP (Figs. 1 and 4), demonstrating that TAM is an uncoupler of oxidative phosphorylation in rat liver mitochondria. Nevertheless, at high concentrations ( $>40 \mathrm{nmol} \mathrm{TAM} / \mathrm{mg}$ protein), it

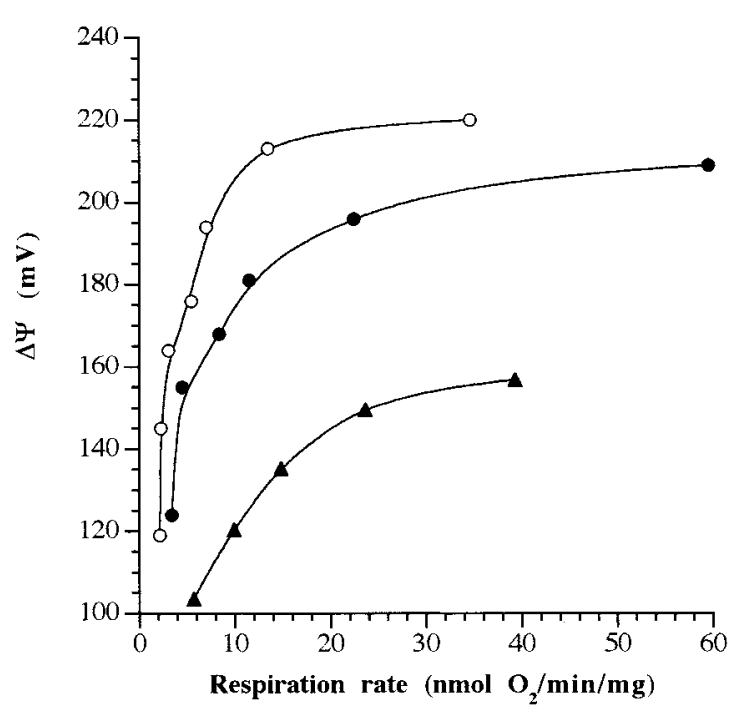

FIG . 6. Effect of TAM on proton leak in nonphosphorylating mitochondria. Nonphosphorylating mitochondria $(1 \mathrm{mg})$ suspended in $1 \mathrm{ml}$ of the standard respiratory medium supplemented with $50 \mathrm{ng} / \mathrm{ml}$ nigericin, $1.5 \mu \mathrm{g} / \mathrm{ml}$ oligomycin, $2 \mu \mathrm{M}$ rotenone, and $4 \mu \mathrm{M} \mathrm{TPP}{ }^{+}$were incubated in the absence $(\bigcirc)$ and in the presence of $20(\bullet)$ and $45(\mathbf{\Delta}) \mathrm{nmol} \mathrm{TAM} / \mathrm{mg}$ protein at $25^{\circ} \mathrm{C} / 3$ min before starting the reactions with $5 \mathrm{mM}$ succinate. Mitochondrial respiration and $\Delta \Psi$ were measured simultaneously with a $\mathrm{TPP}^{+}$-sensitive electrode and a Clark-type $\mathrm{O}_{2}$ electrode, respectively, placed in the same closed reaction chamber. After a steady-state distribution of $\mathrm{TPP}^{+}$, malonate was added to the mitochondria suspension, followed by five further additions. 


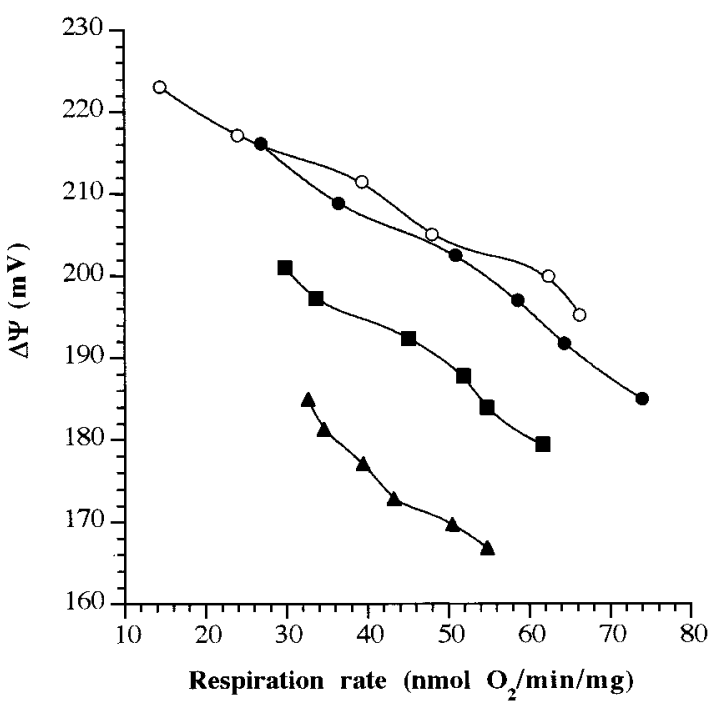

FIG. 7. Effect of TAM on the kinetic response of $\Delta$ p generators to $\Delta \Psi$. Mitochondria were incubated in the absence $(\bigcirc)$ and in the presence of $20(\mathbf{)})$, $40(\boldsymbol{\square})$, and $50(\boldsymbol{\Delta}) \mathrm{nmol} \mathrm{TAM} / \mathrm{mg}$ protein. The experiments were carried out as described in Fig. 6, except that mitochondria were titrated with FCCP.

causes a clear reversion in the stimulation of state 4 respiration, which parallels the rapid collapse of mitochondrial $\Delta \Psi$. Additionally, the approach developed by Brand (1990) corroborates the depressive effect of TAM on FCCP-stimulated respiration and stresses the fact that only high concentrations of TAM ( $\geq 40 \mathrm{nmol} \mathrm{TAM} / \mathrm{mg}$ protein) impair the electron transfer along the respiratory chain (Fig. 7).

The phosphorylation efficiency of mitochondria in the presence of TAM is clearly affected as inferred from either the decrease in the RCR and ADP/O ratios (Fig. 1) or from the fluctuations associated with the phosphorylative cycle induced by ADP (Fig. 2) and the inhibition of state 3 respiration (Fig. 4). It is noteworthy that the oxidative phosphorylation is seriously compromised at concentrations of TAM below those affecting the electron transfer along the respiratory chain. Therefore, in addition to the redox chain, this antiestrogen could affect the rate of ATP synthesis essentially as a consequence of the $\Delta \Psi$ depolarization due to an increase in the proton leak through the mitochondrial inner membrane, since the import of ADP by the adenine nucleotide translocase (ANT) for the phosphorylation and the phosphorylative system use $\Delta \Psi$. Moreover, although we have no direct evidence, the decrease in the phosphorylation efficiency caused by TAM, which induces stimulation of ATPase (Fig. 5), may reflect also a specific inhibition of either the ANT or the phosphate carrier. Therefore, additional experiments currently in progress are required to test these possibilities and to evaluate alterations on cellular energetic charge induced by TAM.

In isolated mitochondria, the state 4 respiration rate is a consequence of the proton permeability of the mitochondrial inner membrane. Therefore, the observed stimulation of state 4 by TAM suggests an increase in the proton leak, pointing out that TAM could act as a proton shuttle or by disrupting the structure of the mitochondrial membrane (Murphy, 1989). The high hydrophobic character and strong partition in biomembranes (Custódio et al., 1991) make it possible for TAM to act as a proton shuttle, since its amine group may bind and translocate protons across the mitochondrial inner membrane. The charged protonated form of tamoxifen may be membrane permeable, thus determining the increase in proton leak induced by this drug and acting like a classic protonophore. This mechanism has been proposed to explain the ability of some amine local anesthetics to uncouple respiration (Garlid and Nakashima, 1983) and it is probable that, at high $\Delta \mathrm{p}$, the stimulation of state 4 respiration induced by low concentrations of TAM could also be explained by this mechanism. However, TAM also determines strong alterations in the membrane structure integrity (Custódio et al., 1996, 1998; Chen et al., 1999; Cruz Silva et al., 2000) that may increase the proton conductance of the mitochondrial inner membrane, leading to the increase in the proton leak by a disrupting rather than by a shuttling mechanism, in agreement with the observations by Chen et al. (1999). Therefore, the observed stimulation of the respiration rate (Figs. 3 and 4) occurs to compensate the depolarization of $\Delta \Psi$, as shown by malonate titration of state 4 respiration (Fig. 6), and both effects are a consequence of the increased proton leak through the mitochondrial inner membrane due to its disruption induced by TAM. Moreover, the inhibition of respiration induced by TAM concentrations above $40 \mu \mathrm{M}$ may be partly due to the disruption of the mitochondrial inner membrane and not to an interaction of TAM with the electron transport chain. Furthermore, the decrease in respiration caused by TAM may reflect other membrane-dependent biological activities of this drug, since TAM, with a $\mathrm{pKa} \sim 8.5$, is a protonated quaternary ammonium cation at the used experimental $\mathrm{pH}$ that partitions strongly in biomembranes (Custódio et al., 1991) and decreases its fluidity (Custódio et al., 1993). As reported for a few lipophilic compounds possessing base- and acid-dissociative groups, with characteristics and effects very similar to those of TAM and also known as uncouplers [e.g., AU-1421 (Nagamune et al., 1993), phloretin (Jonge et al., 1983), amiodarone (Fromenty et al., 1990), local anesthetics (Garlid and Nakashima, 1983), and several nongenotoxic carcinogens (Keller et al., 1992)], the ionizable group of TAM could change the membrane surface potential, thereby changing the interactions between the components of the respiratory chain. Additionally, this drug may decrease the diffusional mobility of membrane proteins, according to its rigidifying effects in biomembranes (Custódio et al., 1993), thereby decreasing the rate of electron transfer along the redox system of mitochondria.

In order to verify the proton leak relationship with membrane disruption induced by TAM, the ATPase activity of intact mitochondria was measured in the presence of TAM and FCCP, followed by the addition of oligomycin (Fig. 5). As described by Chen et al. (1999) for the ATPase activity in 
yeast, we demonstrate that TAM induces ATPase activity of intact mitochondria isolated from rat liver, as also observed for FCCP. The ATPase stimulatory action of FCCP is abolished by oligomycin, in contrast to that induced by high concentrations of TAM (>50 nmol TAM/mg protein). Moreover, the ATPase activity of submitochondrial particles is not significantly affected by TAM (results not shown), indicating that the drug does not act directly on the ATP synthase/ATPase but promotes instead some sort of disruptive effect on the mitochondrial inner membrane. The membrane-disruptive capacity of TAM is also inferred from the decrease in light scattering of nonenergized mitochondria (Custódio et al., 1998), the decrease in the energetic efficiency of the $\mathrm{Ca}^{2+}$ pump of sarcoplasmic reticulum (Custódio et al., 1996), and hemolytic effects (Cruz Silva et al., 2000).

Tamoxifen usually is administrated at a daily dose of 20 $\mathrm{mg} / \mathrm{kg}$ body wt and the range of TAM serum levels is between 50 and $300 \mathrm{ng} / \mathrm{ml}$ (approximately $0.8 \mu \mathrm{M}$ ) (Jordan 1990). However, steady-state tissue concentrations of TAM in rats and humans, including in the hepatic tissue, are 60-70 times higher than in serum (Lien et al., 1991), due to its strong partitioning in biomembranes (Custódio et al., 1991), and at least 4 weeks of administration are required to reach steadystate drug concentrations (Jordan, 1990). Therefore, the estimated drug concentrations in peripheral tissues may reach values (approximately 10 to $50 \mu \mathrm{M}$ ) in the range of our studies.

In conclusion, this work demonstrates that TAM, in the range of concentrations used, which are related with those reached in tissues (Jordan, 1990; Lien et al. 1991; Custódio et al., 1991), may induce substantial alterations on cellular energetic charge as a consequence of the uncoupling of oxidation from phosphorylation, rendering the mitochondria unable to fulfill the cell energy requirements due to the disruption of the mitochondrial inner membrane and by activation of mitochondrial ATPase. These different effects may explain the process of cell death induced by this anticancer agent in different cell types, including ER-negative breast cancer (Jordan 1990), lung adenocarcinoma (Croxtall et al., 1994), prostate cancer (Bergan et al., 1999), ovarian carcinoma (Trope et al., 2000), virus (Laurence et al., 1990), and bacteria (Luxo et al., 1996), since ATP is required to maintain the cell viability. Moreover, our data in isolated mitochondria are according to the $\Delta \Psi$ depolarization induced by TAM either in neurons (Hoyt et al., 2000) or in normal human mammary epithelial cells (Dietze et al., 2001) and may explain the inhibition of $\mathrm{Ca}^{2+}$ uptake by the cardiac sarcoplasmic recticulum (Kargacin et al., 2000) and the TAM-induced inhibition of acidification in ER-independent cells (Altan et al., 1999). The mitochondrial depolarization induced by TAM may be also important as an early event in the promotion of apoptosis, a critical process for normal tissue homeostasis that may be involved in the cytotoxic ER-independent effects of TAM observed in vivo (Dietze et al., 2001). Thus, these proposed ER-independent action mechanisms of TAM, explaining its cytotoxic effects in different cells, raise further questions about the specificity of TAM on breast cancer therapy.

\section{ACKNOWLEDGMENTS}

This work was supported by Sapiens 99/36075/99 research program. Carla M. P. Cardoso is a recipient of a grant from Praxis XXI (Praxis XXI/BD/ 21600/99).

\section{REFERENCES}

Altan, N., Chen, Y., Schindler, M., and Simon, S. M. (1999). Tamoxifen inhibits acidification in cells independent of the estrogen receptor. Proc. Natl. Acad. Sci. USA 96, 4432-4437.

Bergan, R. C., Reed, E., Myers, C. E., Headlee, D., Brawley, O., Cho, H. K., Figg, W. D., Tompkins, A., Linehan, W. M., Kohler, D., Steinberg, S. M., and Blagosklonny, M. V. (1999). A phase II study of high-dose tamoxifen in patients with hormone-refractory prostate cancer. Clin. Cancer Res. 5, 2366-2373.

Brand, M. D. (1990). The proton leak across the mitochondrial inner membrane. Biochim. Biophys. Acta 1018, 128-133.

Brand, M. D., D’Alessandri, L., Reis, H. M.G. P., and Hafner, R. P. (1990). Stimulation of the electron transport chain in mitochondria isolated from rats treated with mannoheptulose or glucagon. Arch. Biochem. Biophys. 283, $278-284$.

Chance, B., and Williams, G. R. (1956). The respiration chain and oxidative phosphorylation. Adv. Enzymol. 17, 65-134.

Charlier, C., Chariot, A., Antoine N., Merville, M., Gielen, J., and Castronovo, V. (1995). Tamoxifen and its active metabolite inhibit growth of estrogen receptor-negative MDA-MB-435 cells. Biochem. Pharmacol. 49, 351-358.

Chen, Y., Schindler, M., and Simon, S. M. (1999). A mechanism for tamoxifen-mediated inhibition of acidification. J. Biol. Chem. 274, 18364-18373.

Coezy, E., Borgna J. L., and Rochefort, H. (1982). Tamoxifen and metabolites in MCF-7 cells: Correlation between binding to estrogen receptor and inhibition of cell growth. Cancer Res. 42, 317-323.

Couldwell, W. T., Weiss, M. H., Degiorgio, C. M., Weimar, L. P., Hinton, D. R., Ehresmsan, G. R., Conti, P. S., Appuzo, M. L. J., Korblith, P., and Detriboler, N. (1993). Clinical and radiographic response in patients with recurrent malignant gliomas treated with high-dose tamoxifen. Neurosurgery 32, 485-490.

Croxtall, J. D., Emmas, C., White, J. O., Choudhary, Q., and Flower, R. J. (1994). Tamoxifen inhibits growth of oestrogen receptor-negative A549 cells. Biochem. Pharmacol. 47, 197-202.

Cruz Silva, M. M., Madeira, V. M. C., Almeida, L. M., and Custódio, J. B. A. (2000). Hemolysis of human erythrocytes induced by tamoxifen is related to disruption of membrane structure. Biochim. Biophys. Acta 1464, 49-61.

Custódio, J. B. A., Almeida, L. M., and Madeira, V. M.C. (1991). A reliable and rapid procedure to estimate drug partitioning in biomembranes. Biochem. Biophys. Res. Commun. 176, 1079-1085.

Custódio, J. B. A., Almeida, L. M., and Madeira, V. M. C. (1993). The anti-cancer drug tamoxifen induces changes in the physical properties of model and native membranes. Biochim. Biophys. Acta 1150, 123-129.

Custódio, J. B. A., Almeida, L. M., and Madeira, V. M. C. (1994). Tamoxifen and hydroxytamoxifen as intramembraneous inhibitors of lipid peroxidation. Evidence for peroxyl radical scavenging activity. Biochem. Pharmacol. 47, 1989-1998.

Custódio, J. B. A., Almeida, L. M., and Madeira, V. M. C. (1996). The effect of the anti-cancer drugs tamoxifen and hydroxytamoxifen on the calcium pump of isolated sarcoplasmic reticulum vesicles. Toxicol. in Vitro 10, 523-531. 
Custódio, J. B. A., Moreno, A. J. M., and Wallace, K. B. (1998). Tamoxifen inhibits induction of the mitochondrial permeability transition by $\mathrm{Ca}^{2+}$ and inorganic phosphate. Toxicol. Appl. Pharmacol. 152, 10-17.

Dietze, E. C., Caldwell, L. E., Grupin, S. L., Mancini, M., and Seewaldt, V. L. (2001).Tamoxifen but not 4-hydroxytamoxifen initiates apoptosis in p53(-) normal human mammary epithelial cells by inducing mitochondrial depolarization. J. Biol. Chem. 276, 5384-5394.

Ferreira, F. M. L., Madeira, V. M. C., and Moreno, A. J. (1997). Interactions of 2,2-bis( $p$-chlorophenyl)-1,1-dichloroethylene with mitochondrial oxidative phosphorylation. Biochem. Pharmacol. 53, 299-308.

Fromenty, D., Fisch, C., Person, A., Letteron, P., Larrey, D., and Pessayre, D. (1990). Dual effect of amiodarone on mitochondrial respiration. Initial protonophoric uncoupling effect followed by inhibition of the respiratory chain at the levels of complex I and complex II. J. Pharmacol. Exp. Ther. 255, 1377-1384.

Fusi, F., Sgaragli, G., and Murphy, M. P. (1992). Interaction of butylated hydroxyanisole with mitochondrial oxidative phosphorylation. Biochem. Pharmacol. 43, 1203-1208.

Garlid, K. D., and Nakashima, R. A. (1983). Studies on the mechanism of uncoupling by amine local anesthetics. Evidence for mitochondrial proton transport mediated by lipophilic ion pairs. J. Biol. Chem. 258, 7974-7980.

Gornall, A. G., Bardawill, C. J., and David, M. M. (1949). Determination of serum proteins by means of the biuret reaction. J. Biol. Chem. 177, 751-766.

Hafner, R. P., Nobes, C. D., McGown, A. D., and Brand, M. D. (1988). Altered relationship between proton motive force and the respiration rate in nonphosphorylating liver mitochondria isolated from rats of different thyroid hormone status. Eur. J. Biochem. 178, 511-518.

Hafner, R. P., Leake, M. J., and Brand, M. D. (1989). Hypothyroidism in rats decreases mitochondrial inner membrane cation permeability. FEBS Lett. 248, 175-178.

Hafner, R. P., Brown, G. C., and Brand, M. D. (1990). Thyroid-hormone control of state 3 respiration in isolated rat liver mitochondria. Biochem. J. 265, 731-734.

Hafner, R. P., and Brand, M. D. (1991). Effect of proton motive force on the relative stoichiometries of the mitochondrial proton pumps. Biochem. J. 275, $75-80$.

Hoyt, K. R., McLaughlin, B. A., Higgins, D. S., and Reynolds, I. J. (2000). Inhibition of glutamate-induced mitochondrial depolarization by tamoxifen in cultured neurons. J. Pharmacol. Exp. Ther. 293, 480-486.

Jonge, P. C., Wiering, T., Putten, J. P. M. V., Krans, M., and Dam, K. V. (1983). Phloretin: An uncoupler and an inhibitor of mitochondrial oxidative phosphorylation. Biochim. Biophys. Acta 722, 219-225.

Jordan, V. C. (1990). Long-term adjuvant tamoxifen therapy for breast cancer. Breast Cancer Res. Treat. 15, 125-136.

Kamo, N., Maratsugu, M., Hongoh, R., and Kobatabe, Y. (1979). Membrane potential of mitochondria measured with an electrode sensitive to tetraphenylphosphonium and relationship between proton electrochemical potential and phosphorylation potential in steady state. J. Membr. Biol. 49, 105-121.

Kargacin, M. E., Ali, Z., Ward, C. A., Pollock, N. S., and Kargacin G. J.
(2000). Tamoxifen inhibits $\mathrm{Ca}^{2+}$ uptake by the cardiac sarcoplasmic reticulum. Eur. J. Biochem. 440, 573-579.

Keller, B. J., Marsman, D. S., Popp, J. A., and Thurman, R. G. (1992). Several nongenotoxic carcinogens uncouple mitochondrial oxidative phosphorylation. Biochim. Biophys. Acta 1102, 237-241.

Laurence, J., Cooke, H., and Sikder, S., K. (1990). Effect of tamoxifen on regulation of viral replication and human immunodeficiency virus (HIV) long terminal repeat-directed transcription in cells chronically infected with HIV-1. Blood 75, 696-703.

Lien, E. A., Solheim, E., and Ueland, P. M. (1991). Distribution of tamoxifen and its metabolites in rat and human tissues during steady-state treatment. Cancer Res. 51, 4837-4844.

Lionetti, L., Iossa, Liverni, S., and Brand, M. D. (1998). Changes in the hepatic mitochondrial respiratory system in the transition from weaning to adulthood in rats. Arch. Biochem. Biophys. 352, 240-246.

Luxo, C., Jurado, A. S., Custódio, J. B. A., and Madeira, V. M. C. (1996). Use of Bacillus stearothermophilus as a model to study tamoxifen-membrane interactions. Toxicol. in Vitro 10, 463-471.

Madeira, V. M. C., Antunes-Madeira, M. C., and Carvalho, A. P. (1974). Activation energies of the ATPase activity of sarcoplasmic reticulum. Biochem. Biophys. Res. Commum. 58, 897-904.

Moreno, A. J., and Madeira, V. M. C. (1991). Mitochondrial bioenergetics as affected by DDT. Biochim. Biophys. Acta 1060, 166-174.

Murphy, M. P. (1989). Slip and leak in mitochondria oxidative phosphorylation. Biochim. Biophys. Acta 977, 123-141.

Murphy, M. P., and Brand, M. D. (1987). The control of the electron flux through cytochrome $c$ oxidase. Biochem. J. 243, 499-505.

Nagamune, K., Fukushima, Y., Takada, J., Yoshida, K., Unami, A., Shimooka, T., and Terada, H. (1993). The lipophilic weak base (Z)-5-methyl-2-[2-(1naphthyl)ethenyl]-4-piperidinopyridine (AU-1421) is a potent protonophore type cationic uncoupler of oxidative phosphorylation in mitochondria. Biochim. Biophys. Acta 1141, 231-237.

Neven, P., and Vernaeve, H. (2000). Guidelines for monitoring patients taking tamoxifen treatment. Drug Saf. 22, 1-11.

Radmacher, M. D., and Simon, R. (2000). Estimation of tamoxifen's efficiency for preventing the formation and growth of breast tumors. J. Natl. Cancer Inst. 92, 48-53.

Sica, G., Natoli, C., Marchetti, P., Piperno, S., and Iacobelli, S. (1984). Tamoxifen induced membrane alterations in human breast cancer cells. $J$. Steroid Biochem. 20, 425-428.

Suwalsky, M., Hernandez, P., Villena F., Aguilar, F., and Sotomayor, C. P. (1998). Interaction of the anticancer drug tamoxifen with the human erythrocyte membrane and molecular models. Z. Naturforsh. Sect. C Biosci. 53, 182-190.

Trope, C. Marth, C., and Kaern, J. (2000). Tamoxifen in the treatment of recurrent ovarian carcinoma. Eur. J. Cancer 36, 59-61.

Wallace, K. B., Eells, J. T., Madeira, V. M. C., Cortopassi, G., and Jones, D. P. (1997). Mitochondria-mediated cell injury. Fundam. Appl. Toxicol. 38, 23-37. 